\title{
Heavy Majorana neutrino pair productions at the LHC in minimal $U(1)$ extended Standard Model
}

\section{Arindam Das}

School of Physics, KIAS, Seoul 02455, Korea E-mail: arindamekias.re.kr

Towards experimental confirmations of the type-I seesaw mechanism, we explore a prospect of discovering the heavy Majorana right-handed neutrinos (RHNs) from a resonant production of a new massive gauge boson $\left(Z^{\prime}\right)$ and its subsequent decay into a pair of RHNs $\left(Z^{\prime} \rightarrow N N\right)$ at the High Luminosity Large Hadron Collider (HL-LHC). Recent simulation studies have shown that the discovery of the RHNs through this process is promising in the future. However, the current LHC data very severely constrains the production cross section of the $Z^{\prime}$ boson into a dilepton final states, $\left(p p \rightarrow Z^{\prime} \rightarrow \ell^{+} \ell^{-}, \ell=e / \mu\right)$. Extrapolating the current bound to the future, we find that a significant enhancement of the branching ratio $\operatorname{BR}\left(Z^{\prime} \rightarrow N N\right)$ over $\operatorname{BR}\left(Z^{\prime} \rightarrow \ell^{+} \ell^{-}\right)$is necessary for the future discovery of RHNs. As a well-motivated simple extension of the standard model (SM) to incorporate the $Z^{\prime}$ boson and the type-I seesaw mechanism, we consider the minimal $U(1)_{X}$ model. We point out that this model can yield a significant enhancement up to $\mathrm{BR}\left(Z^{\prime} \rightarrow N N\right) / \mathrm{BR}\left(Z^{\prime} \rightarrow \ell^{+} \ell^{-}\right) \simeq 5$ (per generation). With such an enhancement and a realistic model-parameter choice to reproduce the neutrino oscillation data, we conclude that the possibility of discovering RHNs with, for example, a $3000 \mathrm{fb}^{-1}$ luminosity implies that the $Z^{\prime}$ boson will be discovered with a luminosity of $853 \mathrm{fb}^{-1}\left(626 \mathrm{fb}^{-1}\right)$ for the normal (inverted) hierarchy of the light neutrino mass pattern.

39th International Conference on High Energy Physics

4-11 July 2018

Seoul, Korea 
Since the RHNs are singlet under the SM gauge group, they can be produced only through their mixings with the SM neutrinos. Particle content under the gauge group $S U(3)_{C} \times S U(2)_{L} \times$ $U(1)_{Y} \times U(1)_{X}: q_{L}^{i}=\left\{\mathbf{3}, \mathbf{2}, \mathbf{1} / \mathbf{6},(\mathbf{1} / \mathbf{6}) \mathbf{x}_{\mathbf{H}}+(\mathbf{1} / \mathbf{3}) \mathbf{x}_{\phi}\right\} ; u_{R}^{i}=\left\{\mathbf{3}, \mathbf{1}, \mathbf{2} / \mathbf{3},(\mathbf{2} / \mathbf{3}) \mathbf{x}_{\mathbf{H}}+(\mathbf{1} / \mathbf{3}) \mathbf{x}_{\phi}\right\} ;$ $d_{R}^{i}=\left\{\mathbf{3}, \mathbf{1},-\mathbf{1} / \mathbf{3},-(\mathbf{1} / \mathbf{3}) \mathbf{x}_{\mathbf{H}}+(\mathbf{1} / \mathbf{3}) \mathbf{x}_{\phi}\right\} ; \ell_{L}^{i}=\left\{\mathbf{1}, \mathbf{2},-\mathbf{1} / \mathbf{2},(-\mathbf{1} / \mathbf{2}) \mathbf{x}_{\mathbf{H}}-\mathbf{x}_{\phi}\right\} ; e_{R}^{i}=\left\{\mathbf{1}, \mathbf{1},-\mathbf{1},-\mathbf{x}_{\mathbf{H}}-\right.$ $\left.\mathbf{x}_{\phi}\right\} ; H=\left\{\mathbf{1}, \mathbf{2},-\mathbf{1} / \mathbf{2},(-\mathbf{1} / \mathbf{2}) \mathbf{x}_{\mathbf{H}}\right\} ; N_{R}^{j}=\left\{\mathbf{1}, \mathbf{1}, \mathbf{0},-\mathbf{x}_{\phi}\right\} ; \Phi=\left\{\mathbf{1}, \mathbf{1}, \mathbf{0},+\mathbf{2} \mathbf{x}_{\phi}\right\}$. Particle content of the minimal $U(1)_{X}$ model, where $i, j=1,2,3$ are the generation indices. Without loss of generality, we fix $x_{\Phi}=1$. Yukawa Interaction : $\mathscr{L}_{Y} \supset-\sum_{i, j=1}^{3} Y_{D}^{i j} \overline{\ell_{L}^{i}} H N_{R}^{j}-\frac{1}{2} \sum_{i=k}^{3} Y_{N}^{k} \Phi \overline{N_{R}^{k c}} N_{R}^{k}+$ h.c., where the first and second terms are the Dirac and Majorana Yukawa couplings. Here we use a diagonal basis for the Majorana Yukawa coupling without loss of generality. After the $\mathrm{U}(1)_{X}$ and the EW symmetry breaking, $\mathrm{U}(1)_{X}$ gauge boson mass, the Majorana masses for the RHNs, and neutrino Dirac masses are generated [4, 5]. Assuming the hierarchy of $\left|m_{D}^{i j} / M_{N}\right| \ll 1$, we have the seesaw formula for the light Majorana neutrinos as $m_{v} \simeq-\frac{1}{M_{N}} m_{D} m_{D}^{T}$, where $M_{N}=m_{N^{1}}=m_{N^{2}}=m_{N^{3}}$. We express the light neutrino flavor eigenstate $(v)$ in terms of the mass eigenstates of the light $\left(v_{m}\right)$ and heavy $\left(N_{m}\right)$ Majorana neutrinos such as $v \simeq U_{\mathrm{MNS}} v_{m}+\mathscr{R} N_{m}$, where $\mathscr{R}=m_{D} / M_{N}$, and $U_{\mathrm{MNS}}$ is the neutrino mixing matrix by which $m_{v}$ is diagonalized as $U_{\mathrm{MNS}}^{T} m_{v} U_{\mathrm{MNS}}=D_{v}=\operatorname{diag}\left(m_{1}, m_{2}, m_{3}\right)$. The heavy neutrino mass eigenstates have the SM gauge bosons as: $\mathscr{L}_{\text {int }} \supset-\frac{g}{\sqrt{2}} W_{\mu}^{+} \overline{\ell_{\alpha}} \gamma^{\mu} P_{L} \mathscr{R}_{\alpha j} N_{m}^{j}-$ $\frac{g}{2 \cos \theta_{\mathrm{W}}} Z_{\mu} \overline{v_{\alpha}} \gamma^{\mu} P_{L} \mathscr{R}_{\alpha j} N_{m}^{j}-\frac{1}{\sqrt{2} v_{h}} h \overline{v_{\alpha}} P_{L} \mathscr{R}_{\alpha j} N_{m}^{j}$ where $\ell_{\alpha}$ and $v_{\alpha}(\alpha=e, \mu, \tau)$ are the three generations of the charged leptons and neutrinos, $P_{L}=\left(1-\gamma_{5}\right) / 2$, and $\theta_{\mathrm{W}}$ is the weak mixing angle. Through the above interactions, a heavy neutrino mass eigenstate $N_{m}^{i}(i=1,2,3)$ decays into $\ell_{\alpha} W, v_{\alpha} Z$, and $v_{\alpha} h,[1,2]$. With the neutrino oscillation data, $M_{N}=\frac{M_{Z}^{\prime}}{4}, M_{Z}^{\prime}=3 \mathrm{TeV}$, we find the maximum value of $\Sigma_{i=1}^{3} B R\left(N N \rightarrow \mu^{ \pm} \mu^{ \pm} W^{\mp} W^{\mp}\right)$ will be $0.210(0.154)$ for the normal (inverted) hierarchy taking lightest neutrino mass eigenvalue as $m_{\text {lightest }}=0.1 \times \sqrt{\Delta m_{12}^{2}}$ and real parameter choice of $O$. We have shown that this model can yield the significant enhancement of $\frac{\operatorname{BR}\left(Z^{\prime} \rightarrow N N\right)}{\operatorname{BR}\left(Z^{\prime} \rightarrow \ell^{+} \ell^{-}\right)} \simeq 3.25$ (per generation) for $x_{H}=-1.2$, with $m_{Z^{\prime}}=3 \mathrm{TeV}$ and $m_{N}=m_{Z^{\prime}} / 4$ therefore $\sigma\left(p p \rightarrow Z^{\prime} \rightarrow N N \rightarrow \mu^{ \pm} \mu^{ \pm} W^{\mp} W^{\mp}\right) \simeq 0.02 \mathrm{fb}$ for the 5- $\sigma$ discovery of the RHN with a $3000 \mathrm{fb}^{-1}$ luminosity [3] when $Z^{\prime}$ can be discovered at 853 (626) $\mathrm{fb}^{-1}$ luminosity for the normal (inverted) hierarchical light neutrino mass pattern.

\section{References}

[1] A. Das, P. Konar and S. Majhi, "Production of Heavy neutrino in next-to-leading order QCD at the LHC and beyond,” JHEP 1606, 019 (2016) doi:10.1007/JHEP06(2016)019 [arXiv:1604.00608 [hep-ph]].

[2] F. F. Deppisch, P. S. Bhupal Dev and A. Pilaftsis, "Neutrinos and Collider Physics," New J. Phys. 17, no. 7, 075019 (2015) doi:10.1088/1367-2630/17/7/075019 [arXiv:1502.06541 [hep-ph]].

[3] P. Cox, C. Han and T. T. Yanagida, "LHC Search for Right-handed Neutrinos in $Z$ ' Models," JHEP 1801, 037 (2018) doi:10.1007/JHEP01(2018)037 [arXiv:1707.04532 [hep-ph]].

[4] A. Das, N. Okada and D. Raut, "Enhanced pair production of heavy Majorana neutrinos at the LHC," Phys. Rev. D 97, no. 11, 115023 (2018) doi:10.1103/PhysRevD.97.115023 [arXiv:1710.03377 [hep-ph]].

[5] A. Das, N. Okada and D. Raut, "Heavy Majorana neutrino pair productions at the LHC in minimal U(1) extended Standard Model,” Eur. Phys. J. C 78, no. 9, 696 (2018) doi:10.1140/epjc/s10052-018-6171-8 [arXiv:1711.09896 [hep-ph]]. 